\title{
Delay Dependent Anti-windup Synthesis for Time-varying Delay Systems with Saturating Actuators
}

\author{
N. El Fezazi F. El Haoussi $\quad$ E.H. Tissir \\ Lessi, Department of Physics \\ Faculty of Sciencs Dhar El Mehraz \\ BP 1796, Fes-Atlas, Morocco
}

\author{
F. Tadeo \\ Dpto. Ingenieria de Sistemas y Automatica \\ Universidad de Valladolid \\ 47005 Valladolid, Spain
}

\begin{abstract}
The synthesis of an anti-windup compensator for systems with time-varying delays is solved in this paper. More precisely, LMI conditions are proposed that guarantee the stability of the closedloop system, with a enlarged domain of attraction. An ellipsoid and a polyhedral set are used to bound this domain of attraction, making possible to derive a new sector condition. In addition, an algorithm is provided to optimize the anti-windup gain, reducing the conservatism. Numerical examples illustrate the effectiveness of our methodology, which improves previous works.
\end{abstract}

\section{General Terms:}

Time-varying delay, anti-windup

\section{Keywords:}

Actuator saturation, LMI, time-varying delay, anti-windup, region of stability

\section{INTRODUCTION}

Time delays are a common phenomena in many physical systems (for example, in mechanical, communication and chemical process). The simultaneous presence of delays and control saturations are the cause of performance degradation and eventual instability. Many methods have been proposed to address the stability of linear time-delay systems [4 8, 7, 15], including the stabilization with saturating actuators [10, 1, 5, 12].

This paper concentrates on anti-windup methodologies to stabilize time-delay systems in the presence of control constraints: in this context we can just cite [2, 14, 13]. The anti-windup compensation is known to be a efficient technique to cope with undesirable effects (on performance and stability) produced by actuator saturation in control loops. The basic idea underlining anti-windup designs is that when the control saturates, it is temporarily modified using an anti-windup compensator in order to recover, as much as possible, the performance expected on the basis of the unsaturated system. Motivated by this, we consider $\mathcal{L}_{2}$-gain analysis and anti-windup compensation gains design for linear systems subject to timevarying delay and saturating actuators. The method is based on the Lyapunov-Krasovskii (L-K) approach, which allows to obtain the conditions directly in an LMI form.

The plan of the paper is as follows: Section 2 presents the prob- lem statement, and some preliminary results. In section 3 we derive a result for stabilization anti-windup gain computation using the Lyapunov-Krasovskii functional. Section 4 presents some convex optimization problems, based on the statements of Section 5. Some examples are solved in section 6 to illustrate the proposed solution. In last section 6 we will give some conclusions.

Notation: Throughout the paper the superscript ' $T$ ' stands for matrix transposition, $\Re^{n}$ denotes the $n$ dimensional Euclidean space with vector norm $\|\|,. \Re^{n \times m}$ is the set of all $n \times m$ real matrices, and the notation $P>0$, for $P \in \Re^{n \times n}$ means that $P$ is symmetric and positive definite. The space of the continuously differentiable vector functions $\phi$ over $[-h, 0]$ is denoted by $C^{1}[-h, 0] . A_{(i)}$ denotes the $i$ th row of matrix. For two symmetric matrices, $A$ and $B, A \geq B$ (respectively $A>B$ ) means that $A-B$ is positive semi-definite (respectively positive definite). $\bar{\lambda}(P)$ and $\underline{\lambda}(P)$ denote, respectively, the maximal and minimal eigenvalues of a matrix $P$. I denotes the identity matrix of appropriate dimensions. * denotes symmetric block elements in a matrix.

\section{PROBLEM FORMULATION AND PRELIMINARIES}

In this paper we are interested in the following linear time-delay system:

$$
\begin{aligned}
\dot{x}(t) & =A x(t)+A_{h} x(t-h(t))+B u(t)+B_{w} w(t) \\
y(t) & =C_{y} x(t) \\
z(t) & =C_{z} x(t)+D_{z} u(t)
\end{aligned}
$$

where $x(t) \in \Re^{n}, u(t) \in \Re^{m}, w(t) \in \Re^{q}, y(t) \in \Re^{p}$ and $z(t) \in \Re^{p}$ are the plant state, input, disturbance, measured output and regulated output, respectively, with $A, A_{h}, B, B_{w}, C_{y}, C_{z}$ and $D_{z}$ known constant real matrices. The delay $h(t)$ is assumed to be an unknown but bounded function of time, continuously differentiable, with their rate of change bounded as follows:

$$
0 \leq h(t) \leq h_{m} \quad, \quad \dot{h}(t) \leq d
$$

where $h_{m}>0, d<1$ are given positive constants (these bound are strictly smaller than one to ensure causality: see [4]).

The initial condition of system (1) is given by:

$$
x(\theta)=\phi(\theta), \theta \in\left[-h_{m}, 0\right]
$$

where $\phi($.$) is a vector of differentiable functions of initial values$ (i.e., $\phi \in \mathbf{C}^{1}\left[-h_{m}, 0\right]$ ). 
The control input is supposed to be bounded as follows:

$$
-u_{0(i)} \leq u_{(i)}(t) \leq u_{0(i)}, \quad u_{0(i)}>0, \quad i=1, \ldots, m
$$

The disturbance vector $w(t)$ is assumed to be limited in energy, that is, $w(t) \in \mathcal{L}_{2}$. Hence, for some scalar $\delta, 0 \leq \frac{1}{\delta} \leq \infty$, the disturbance $w(t)$ is bounded as follows

$$
\|w(t)\|_{2}^{2}=\int_{0}^{\infty} w^{T}(t) w(t) d t \leq \frac{1}{\delta}
$$

Considering system (1), we assume that the dynamic output stabilizing compensator is written in the form:

$$
\begin{aligned}
& \dot{x}_{c}(t)=A_{c} x_{c}(t)+B_{c} y(t) \\
& y_{c}(t)=C_{c} x_{c}(t)+D_{c} y(t)
\end{aligned}
$$

Where $x_{c}(t) \in \Re^{n_{c}}$ is the controller state, $u_{c}(t)=y(t) \in \Re^{p}$ is the controller input, and $y_{c}(t) \in \Re^{m}$ is the controller output. This compensator is designed to guarantee the requirements of performance and stability of the closed-loop system in the absence of the control saturation.

$A_{c}, B_{c}, C_{c}$ and $D_{c}$ are known constant real matrices of appropriate dimensions.

In the presence of actuator saturation, the control signal of the system can be described as

$$
u(t)=\operatorname{sat}\left(y_{c}(t)\right)
$$

where $\operatorname{sat}\left(y_{c(i)}(t)\right)=\operatorname{sign}\left(y_{c(i)}(t)\right) \min \left\{\left|y_{c(i)(t)}\right|, u_{0(i)}\right\}, i=$ $1, \ldots, m$

The anti-windup compensator $E_{c} \psi\left(y_{c}(t)\right), \quad E_{c} \in \Re^{n_{c} \times m}$ is proposed to mitigate the undesirable effects of windup, caused by the control saturation. This anti-windup generates a signal that is added to the control signal. Thus, the modified compensator has the form

$$
\begin{aligned}
& \dot{x}_{c}(t)=A_{c} x_{c}(t)+B_{c} y(t)-E_{c} \psi\left(y_{c}(t)\right) \\
& y_{c}(t)=C_{c} x_{c}(t)+D_{c} y(t)
\end{aligned}
$$

The compensator input is given by the vector valued dead zone nonlinearity $\psi\left(y_{c}(t)\right)$, which is obtained as the difference between the applied control signal and the controller output signal; that is,

$$
\psi\left(y_{c}\right)=y_{c}(t)-\operatorname{sat}\left(y_{c}\right)(t)
$$

If we use a dead zone for the compensated dynamic linear controller, we get the following augmented system:

$$
\begin{aligned}
\dot{\xi}(t)= & \mathbb{A} \xi(t)+\mathbb{A}_{h} \xi(t-h(t))-\left(\mathbb{B}+\mathbb{R} E_{c}\right) \psi(\mathbb{K} \xi(t)) \\
& +\mathbb{B}_{w} w(t) \\
z(t)= & \mathbb{C}_{z} \xi(t)-\mathbb{D}_{z} \psi(\mathbb{K} \xi(t))
\end{aligned}
$$

Where $\xi(t)=\left[\begin{array}{c}x(t) \\ x_{c}(t)\end{array}\right], \mathbb{K}=\left[\begin{array}{ll}D_{c} C_{y} C_{c}\end{array}\right], \mathbb{A}=$ $\left[\begin{array}{cc}A+B D_{c} C_{y} & B C_{c} \\ B_{c} C_{y} & A_{c}\end{array}\right], \mathbb{A}_{h}=\left[\begin{array}{cc}A_{h} & 0 \\ 0 & 0\end{array}\right], \mathbb{B}=\left[\begin{array}{c}B \\ 0\end{array}\right]$, $\mathbb{R}=\left[\begin{array}{c}0 \\ I_{n_{c}}\end{array}\right], \mathbb{B}_{w}=\left[\begin{array}{c}B_{w} \\ 0\end{array}\right]$

$\mathbb{C}_{z}=\left[\begin{array}{ll}C_{z}+D_{z} D_{c} C_{y} & D_{z} C_{c}\end{array}\right]$ and $\mathbb{D}_{z}=D_{z}$.

With initial conditions $\phi_{\xi}$ defined on $\left[-h_{m}, 0\right]$, i.e., $\phi_{\xi}=\xi(\theta)$, $\theta=\left[-h_{m}, 0\right]$.

Consider a matrix $\mathbb{G} \in \Re^{m \times\left(n+n_{c}\right)}$ and define the following polyhedral set

$$
\mathcal{S}=\left\{\xi \in \Re^{n+n_{c}} ;\left|\left(\mathbb{K}_{(i)}-\mathbb{G}_{(i)}\right) \xi(t)\right| \leq u_{0(i)}, i=1, \ldots, m\right\}
$$

LEMma 1. [13]. Consider now the dead-zone nonlinearity $\psi(\mathbb{K} \xi(t)):$ If $\xi(t) \in \mathcal{S}$, then the relation

$$
\psi(\mathbb{K} \xi(t))^{T} T_{0}[\psi(\mathbb{K} \xi(t))-\mathbb{G} \xi(t)] \leq 0
$$

The first problem solved in this paper is then stated as follows:

PROBLEM 1. Given $h_{m}$, synthesize an the anti-windup compensator that simultaneously ensures the $\mathcal{L}_{2}$ input-to-state stability and the internal stability of the closed-loop system.

More explictely, to solve Problem 1 the aim is to find a stabilizing $E_{c}$ that maximizes the size of the domain of attraction for the closed-loop system $[10$.

\section{MAIN RESULTS}

In this section we derive a result for solving Problem 1. We first give some sufficient conditions for the system (10) to be asymptotically stable:

LEMMA 2. The system (10) is asymptotically stable if there exist $P=P^{T}>0, Q=Q^{T}>0$ and $R=R^{T}>0$, a positive definite diagonal matrix $T_{0} \in \Re^{m \times m}$ and appropriately dimensioned matrices $N_{1}, N_{2}, T_{1}$, and $T_{2}$ such that the following condition holds:

$$
\left[\begin{array}{ccccccc}
\Pi_{11} & \Pi_{12} & \Pi_{13} & \Pi_{14} & T_{1} \mathbb{B}_{w} & h_{m} N_{1} & \mathbb{C}_{z}^{T} \\
* & \Pi_{22} & \Pi_{23} & \Pi_{24} & T_{2} \mathbb{B}_{w} & 0 & 0 \\
* & * & \Pi_{33} & 0 & 0 & h_{m} N_{2} & 0 \\
* & * & * & \Pi_{44} & 0 & 0 & -\mathbb{D}_{z}^{T} \\
* & * & * & * & -I & 0 & 0 \\
* & * & * & * & * & -h_{m} R & 0 \\
* & * & * & * & * & * & -\gamma I
\end{array}\right]<0,
$$

where

$$
\begin{aligned}
& \Pi_{11}=T_{1} \mathbb{A}+\mathbb{A}^{T} T_{1}^{T}+Q+N_{1}+N_{1}^{T} \\
& \Pi_{12}=P-T_{1}+\mathbb{A}^{T} T_{2}^{T} \\
& \Pi_{22}=-T_{2}-T_{2}^{T}+h_{m} R \\
& \Pi_{13}=-N_{1}+N_{2}^{T}+T_{1} \mathbb{A}_{h} \\
& \Pi_{23}=T_{2} \mathbb{A}_{h} \\
& \Pi_{33}=-N_{2}-N_{2}^{T}-(1-d) Q \\
& \Pi_{14}=-T_{1}\left(\mathbb{B}+\mathbb{R} E_{c}\right)+G^{T} T_{0} \\
& \Pi_{24}=-T_{2}\left(\mathbb{B}+\mathbb{R} E_{c}\right) \\
& \Pi_{44}=-2 T_{0}
\end{aligned}
$$

Proof. Considering the Lyapunov-Krasovskii functional candidate

$$
\begin{aligned}
V(t)= & \xi^{T}(t) P \xi(t)+\int_{t-h(t)}^{t} \xi^{T}(s) Q \xi(s) d s \\
& +\int_{-h_{m}}^{0} \int_{t+\theta}^{t} \dot{\xi}^{T}(s) R \dot{\xi}(s) d s d \theta
\end{aligned}
$$

where $P=P^{T}>0, Q=Q^{T}>0$ and $R=R^{T}>0$.

Calculating the time derivative of the proposed Lyapunov function 
along the trajectory of the system 10$]$ and using 22 yields:

$$
\begin{aligned}
\dot{V}(t) \leq & 2 \xi^{T}(t) P \dot{\xi}(t)+\xi^{T}(t) Q \xi(t) \\
& -(1-d) \xi^{T}(t-h(t)) Q \xi(t-h(t)) \\
& +h_{m} \dot{\xi}^{T}(t) R \dot{\xi}(t)-\int_{t-h_{m}}^{t} \dot{\xi}^{T}(s) R \dot{\xi}(s) d s
\end{aligned}
$$

From (2), it is clear that the following is true:

$$
-\int_{t-h_{m}}^{t} \dot{\xi}^{T}(s) R \dot{\xi}(s) d s \leq-\int_{t-h(t)}^{t} \dot{\xi}^{T}(s) R \dot{\xi}(s) d s
$$

For any $N_{1}, N_{2}$, applying the Lemma in [8] gives the following integral inequality:

$$
\begin{aligned}
-\int_{t-h(t)}^{t} \dot{\xi}^{T}(s) R \dot{\xi}(s) d s \leq & 2\left[\begin{array}{ll}
\xi(t)^{T} & \xi(t-h(t))^{T}
\end{array}\right] \\
& \times\left[\begin{array}{ll}
N_{1} & -N_{1} \\
N_{2} & -N_{2}
\end{array}\right]\left[\begin{array}{c}
\xi(t) \\
\xi(t-h(t))
\end{array}\right] \\
& +h_{m}\left[\begin{array}{ll}
\xi(t)^{T} & \xi(t-h(t))^{T}
\end{array}\right]\left[\begin{array}{c}
N_{1} \\
N_{2}
\end{array}\right] \\
& \times R^{-1}\left[\begin{array}{ll}
N_{1}^{T} & N_{2}^{T}
\end{array}\right]\left[\begin{array}{c}
\xi(t) \\
\xi(t-h(t))
\end{array}\right]
\end{aligned}
$$

Using the free weighting matrix approach introduced in [9], for appropriately matrices $T_{1}$ and $T_{2}$ we have that:

$$
\begin{aligned}
& 2\left[\xi^{T}(t) T_{1}+\dot{\xi}^{T}(t) T_{2}\right] \times\left[-\dot{\xi}(t)+\mathbb{A} \xi(t)+\mathbb{A}_{h} \xi(t-h(t))\right. \\
& \left.-\left(\mathbb{B}+\mathbb{R E}_{c}\right) \psi(\mathbb{K} \xi(t))+\mathbb{B}_{w} w(t)\right]=0
\end{aligned}
$$

For a prescribed scalar $\gamma$, we define the auxiliary function

$$
J(t)=\dot{V}(t)-w^{T}(t) w(t)+\frac{1}{\gamma} z^{T} z(t)
$$

It follows that:

$$
\begin{aligned}
& J(t) \leq 2 \xi^{T}(t) P \dot{\xi}(t)+\xi^{T}(t) Q \xi(t) \\
& -(1-d) \xi^{T}(t-h(t)) Q \xi(t-h(t))+h_{m} \dot{\xi}^{T}(t) R \dot{\xi}(t)
\end{aligned}
$$

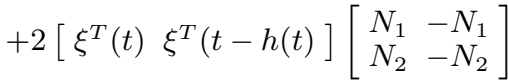

$$
\begin{aligned}
& \times\left[\begin{array}{c}
\xi(t) \\
\xi(t-h(t))
\end{array}\right]+h_{m}\left[\begin{array}{ll}
\xi(t)^{T} & \xi(t-h(t))^{T}
\end{array}\right] \\
& \times\left[\begin{array}{c}
N_{1} \\
N_{2}
\end{array}\right] R^{-1}\left[\begin{array}{ll}
N_{1}^{T} & N_{2}^{T}
\end{array}\right]\left[\begin{array}{c}
\xi(t) \\
\xi(t-h(t))
\end{array}\right] \\
& +2\left[\xi^{T}(t) T_{1}+\dot{\xi}^{T}(t) T_{2}\right] \times[-\dot{\xi}(t)+\mathbb{A} \xi(t) \\
& \left.+\mathbb{A}_{h} \xi(t-h(t))-\left(\mathbb{B}+\mathbb{R E}_{c}\right) \psi(\mathbb{K} \xi(t))+\mathbb{B}_{w} w(t)\right] \\
& -2 \psi(\mathbb{K} \xi(t))^{T} T_{0}[\psi(\mathbb{K} \xi(t))-G \xi(t)] \\
& -w^{T}(t) w(t)+\frac{1}{\gamma} z^{T}(t) z(t)
\end{aligned}
$$

Thus, by simple manipulation the inequality 20, can be rewritten as follows:

$$
\begin{aligned}
& J(t) \leq \eta^{T}(t) \Pi \eta(t)+h_{m}\left[\xi^{T}(t) \xi^{T}(t-h(t))\right] \\
& \times\left[\begin{array}{l}
N_{1} \\
N_{2}
\end{array}\right] R^{-1}\left[\begin{array}{ll}
N_{1}^{T} & N_{2}^{T}
\end{array}\right]\left[\begin{array}{c}
\xi(t) \\
\xi(t-h(t))
\end{array}\right]
\end{aligned}
$$

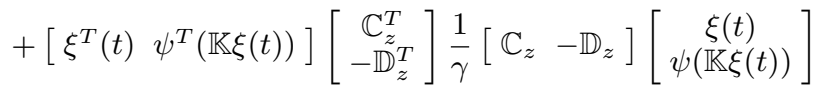

with

$$
\begin{gathered}
\eta^{T}(t)=\left[\begin{array}{lllll}
\xi^{T}(t) & \dot{\xi}^{T}(t) & \xi^{T}(t-h(t)) & \psi^{T}(\mathbb{K} \xi(t) & \left.w^{T}(t)\right)
\end{array}\right], \\
\Pi=\left[\begin{array}{ccccc}
\Pi_{11} & \Pi_{12} & \Pi_{13} & \Pi_{14} & T_{1} \mathbb{B}_{w} \\
* & \Pi_{22} & \Pi_{23} & \Pi_{24} & T_{2} \mathbb{B}_{w} \\
* & * & \Pi_{33} & 0 & 0 \\
* & * & * & \Pi_{44} & 0 \\
* & * & * & * & -I
\end{array}\right]
\end{gathered}
$$

and $\Pi_{i, j},(i, j)=(1,1),(1,2), \ldots,(4,4)$ are defined previously. Since 13 holds, then, by Schur complement, $J(t)$ is negative definite, which ensures the asymptotic stability of the system (10).

Now, we provide the conditions that satisfy the objective of the Problem 1 defined in Section 2.

THEOREM 3. If there exist symmetric positive-definite matrices $\bar{P}, \bar{Q}, \bar{R} \in \Re^{\left(n+n_{c}\right) \times\left(n+n_{c}\right)}$, a diagonal positive-definite matrix $S \in \Re^{m \times m}$, matrices $X_{1}, \bar{N}_{1}, \bar{N}_{2} \in \Re^{\left(n+n_{c}\right) \times\left(n+n_{c}\right)}, W \in$ $\Re^{m \times\left(n+n_{c}\right)}, Y_{c} \in \Re^{m \times n_{c}}$, and positive scalars $\gamma, \mu$ and $\alpha$ satisfying the following conditions:

$$
\left[\begin{array}{ccccccc}
\Sigma_{11} & \Sigma_{12} & \Sigma_{13} & \Sigma_{14} & \mathbb{B}_{w} & h_{m} \bar{N}_{1} & X_{1} \mathbb{C}_{z}^{T} \\
* & \Sigma_{22} & \Sigma_{23} & \Sigma_{24} & \alpha \mathbb{B}_{w} & 0 & 0 \\
* & * & \Sigma_{33} & 0 & 0 & h_{m} \bar{N}_{2} & 0 \\
* & * & * & -2 S & 0 & 0 & -S \mathbb{D}_{z}^{T} \\
* & * & * & * & -I & 0 & 0 \\
* & * & * & * & * & -h_{m} \bar{R} & 0 \\
* & * & * & * & * & * & -\gamma I
\end{array}\right]<0
$$

$$
\left[\begin{array}{cc}
\bar{P} & X_{1} \mathbb{K}_{(i)}^{T}-W_{(i)}^{T} \\
* & \mu u_{0(i)}^{2}
\end{array}\right] \geq 0, \quad i=1, \ldots, m
$$

$$
\mu-\delta \leq 0
$$

where

$$
\begin{aligned}
& \Sigma_{11}=\mathbb{A} X_{1}^{T}+X_{1} \mathbb{A}^{T}+\bar{Q}+\bar{N}_{1}+\bar{N}_{1}^{T} \\
& \Sigma_{12}=\bar{P}-X_{1}^{T}+\alpha X_{1} \mathbb{A}^{T} \\
& \Sigma_{22}=-\alpha X_{1}^{T}-\alpha X_{1}+h_{m} \bar{R} \\
& \Sigma_{13}=-\bar{N}_{1}+\bar{N}_{2}^{T}+\mathbb{A}_{h} X_{1}^{T} \\
& \Sigma_{23}=\alpha \mathbb{A}_{h} X_{1}^{T} \\
& \Sigma_{33}=-\bar{N}_{2}-\bar{N}_{2}^{T}-(1-d) \bar{Q} \\
& \Sigma_{14}=-\left(\mathbb{B} S+\mathbb{R} Y_{c}\right)+W^{T} \\
& \Sigma_{24}=-\alpha\left(\mathbb{B} S+\mathbb{R} Y_{c}\right)
\end{aligned}
$$

then the anti-windup gain $E_{c}=Y_{c} S^{-1}$ ensures that: 
(1) the trajectories of system (10) are bounded for every initial condition satisfying

$$
\begin{gathered}
\beta=\left[\bar{\lambda}\left(X_{1}^{-1} \bar{P} X_{1}^{-T}\right)+h_{m} \bar{\lambda}\left(X_{1}^{-1} \bar{Q} X_{1}^{-T}\right)\right]\left\|\phi_{\xi}\right\|_{c}^{2} \\
+\frac{h_{m}^{2}}{2} \bar{\lambda}\left(X_{1}^{-1} \bar{R} X_{1}^{-T}\right)\left\|\dot{\phi}_{\xi}\right\|_{c}^{2} \leq \mu^{-1}-\delta^{-1}
\end{gathered}
$$

(2)

$$
\|z\|_{2}^{2} \leq \gamma\|w\|_{2}^{2}+\gamma V(0)
$$

(3) when $w(t)=0$, for all initial conditions satisfying $\beta \leq \mu^{-1}$ the corresponding trajectories converge asymptotically to the origin.

Proof. From the functional (14), it follows that

$$
\begin{aligned}
V(0)= & \xi^{T}(0) P \xi(0)+\int_{-h_{m}}^{0} \xi^{T}(s) Q x(s) d s \\
& +\int_{-h_{m}}^{0} \int_{\theta}^{0} \dot{\xi}^{T}(s) R \dot{\xi}(s) d s d \theta \\
& \leq\left[\bar{\lambda}(P)+h_{m} \bar{\lambda}(Q)\right]\left\|\phi_{\xi}\right\|_{c}^{2}+\frac{h_{m}^{2}}{2} \bar{\lambda}(R)\left\|\dot{\phi}_{\xi}\right\|_{c}^{2}
\end{aligned}
$$

From [19], if $J(t)<0$, it follows that

$$
\begin{aligned}
\int_{0}^{T} J(t) d t= & V(T)-V(0)-\int_{0}^{T} w^{T}(t) w(t) d t \\
& +\frac{1}{\gamma} \int_{0}^{T} z^{T}(t) z(t) d t<0
\end{aligned}
$$

which implies that

$$
V(T) \leq V(0)+\|w(t)\|_{2}^{2} \leq \beta+\delta^{-1} \leq \mu^{-1} .
$$

$\forall w(t)$ satisfying $\left[5\right.$, and $\forall \phi_{\xi}$ such that $\beta+\delta^{-1} \leq \mu^{-1}$

Hence, one gets $\xi^{T}(T) P \xi(T) \leq V(T) \leq \mu^{-1}$; that is, for all $T>0$ the trajectories of the system do not leave the set $\varepsilon\left(P, \mu^{-1}\right)=\left\{\xi(t) \in \Re^{n+n_{c}} ; \xi^{T}(t) P \xi(t) \leq \mu^{-1}\right\}$.Moreover, for $T \rightarrow \infty, 28$ yields $\|z\|_{2}^{2} \leq \gamma\|w\|_{2}^{2}+\gamma V(0)$.

In the sequel, we show that the fulfillment of (22)-(24) implies that $J<0$, provided that $\phi_{\xi}$ is such that $\beta+\delta^{-1} \leq \mu^{-1}$ and $w(t)$ satisfies [5].

Take $T_{2}=\alpha T_{1}$, where $\alpha$ is a scalar tuning parameter. Then multiplying the both sides of $(13)$ by $\Delta$ and $\Delta^{T}$, on the left and on the right, respectively, with $\Delta=\operatorname{diag}\left\{T_{1}^{-1}, T_{1}^{-1}, T_{1}^{-1}, T_{0}^{-1}, I, T_{1}^{-1}, I\right\}$, and introducing some changes of variables such that:

$$
\begin{aligned}
& X_{1}=T_{1}^{-1}, \bar{P}=X_{1} P X_{1}^{T}, \bar{N}_{1}=X_{1} N_{1} X_{1}^{T}, \\
& \bar{N}_{2}=X_{1} N_{2} X_{1}^{T}, \bar{Q}=X_{1} Q X_{1}^{T}, \bar{R}=X_{1} R X_{1}^{T}, \\
& Y_{c}=E_{c} T_{0}^{-1}, S=T_{0}^{-1}, W=G X_{1}^{T} .
\end{aligned}
$$

Thus, we obtain the inequalities 22] of Theorem 1.

On the other hand, if

$$
\left[\begin{array}{cc}
P & \mathbb{K}_{(i)}^{T}-G_{(i)}^{T} \\
* & \mu u_{0(i)}^{2}
\end{array}\right] \geq 0, \quad i=1, \ldots, m
$$

It follows that $\varepsilon\left(P, \mu^{-1}\right) \subset \mathcal{S}\left(u_{0}\right)$. As in [2], by multiplying on the left by $\Delta=\operatorname{diag}\left[X_{1}, I\right]$ and on the right by $\Delta^{T}=\operatorname{diag}\left[X_{1}^{T}, I\right]$, we obtain the LMI $(23)$. Hence, LMI $(23)$ ensures that $\varepsilon\left(P, \mu^{-1}\right) \subset$ $\mathcal{S}\left(u_{0}\right)$. This fact enforces the validity of the sector condition 12 .
The simultaneous verification of 22)-(24) ensures that $J(t)<0$ $\forall w(t)$ such that $\|w(t)\|_{2}^{2} \leq \frac{1}{\delta}$, and for all initial condition $\phi_{\xi}$. This concludes the proof of the first and second items of Theorem 1. Consider now $w(t)=0$. Then, $J(t)<0$ implies that $\dot{V}(t)<$ $-\frac{1}{\gamma} z^{T}(t) z(t)<0$, provided that $\xi(t) \in \mathcal{S}$. Hence, from 27 if $\phi_{\xi}$ is such that $\beta<\mu^{-1}$, we have

$$
\xi^{T}(t) P \xi(t) \leq V(t) \leq V(0) \leq \beta \leq \mu^{-1}
$$

which means that we get $\xi(t) \in \varepsilon\left(P, \mu^{-1}\right)$, for all $t \geq 0$. Because the LMI 23] is satisfied, it follows that $\xi(t) \in \mathcal{S}$, for all $t \geq 0$. Thus, for any initial condition $\beta \leq \mu^{-1}$ it follows that $V(t)<0$, which concludes the proof of the third item of Theorem 1 .

REMARK 1. In the proof of Theorem 1, the use of free matrices $N_{1}, N_{2}, T_{1}$ and a scalar tuning parameter $\alpha$ provides more freedom for search the compensation gain $E_{c}$, to ensure that the initial conditions of the closed-loop system and the disturbances belong to certain admissible sets.

\section{OPTIMIZATION PROBLEMS}

The proposed conditions in Theorem 1 can be cast into a convex optimization problem to compute the compensation gain $E_{c}$ which ensures that the state trajectory of the closed-loop system (10) starting from the origin will remain inside a bounded set for any disturbance satisfying (5) and minimize the upper bound of the restricted $\mathcal{L}_{2}-$ gain

The idea is to maximize the $\mathcal{L}_{2}$ norm bound on the disturbance for which it can be ensured that the system trajectories remain bounded. Considering that the initial condition is null (i.e. $\left.\phi \in \mathbf{C}^{1}[-h, 0]\right)$ this can be formalized as follows:

$$
\begin{aligned}
& \min \mu \\
& \text { subject to } 22-23
\end{aligned}
$$

For a non-null bound on the $\mathcal{L}_{2}$ norm of the admissible disturbances (given by $\mu^{-1}=\delta^{-1}$ ), the idea is to minimize the upper bound for the $\mathcal{L}_{2}$ gain of $w(t)$ on $z(t)$. Considering that the initial condition is null, this can be obtained from the solution of the following convex optimization problems

$$
\begin{aligned}
& \min \gamma \\
& \text { subject to } 22-24
\end{aligned}
$$

We consider now the disturbance free case $\omega(t)=0$. The synthesis objective regards therefore the determination of a controller which leads to a set of admissible initial conditions as large as possible, that verify the condition 25 with $\beta \leq \mu^{-1}$.

Consider $\left\|\phi_{\xi}\right\|_{c}^{2}=\kappa_{1}$ and $\left\|\dot{\phi}_{\xi}\right\|_{c}^{2}=\kappa_{2}$. The maximisation of the region of stability of the closed-loop system can be done by imposing the conditions on the maximal eigenvalues of $X_{1}^{-1} \bar{P} X_{1}^{-T}$, $X_{1}^{-1} \bar{Q} X_{1}^{-T}$ and $X_{1}^{-1} \bar{R} X_{1}^{-T}$.

These conditions can be written as follows:

$$
\begin{aligned}
& {\left[\begin{array}{cc}
\sigma_{1} I & X_{1}^{-1} \\
X_{1}^{-T} & \bar{P}^{-1}
\end{array}\right] \geq 0,\left[\begin{array}{cc}
\sigma_{2} I & X_{1}^{-1} \\
X_{1}^{-T} & \bar{Q}^{-1}
\end{array}\right] \geq 0,} \\
& {\left[\begin{array}{cc}
\sigma_{3} I & X_{1}^{-1} \\
X_{1}^{-T} & \bar{R}^{-1}
\end{array}\right] \geq 0 .}
\end{aligned}
$$


Therefore, one gets

$$
\left[\sigma_{1}+h_{m} \sigma_{2}\right] \kappa_{1}+\frac{h_{m}^{2}}{2} \sigma_{3} \kappa_{2} \leq \mu^{-1}-\delta^{-1} .
$$

Let us introduce the new matrix variables:

$$
X_{1}^{-1}=\widetilde{X}_{1}, \bar{P}^{-1}=\widetilde{P}, \bar{Q}^{-1}=\widetilde{Q} \text { and } \bar{R}^{-1}=\widetilde{R}
$$

The conditions 34 can be replaced by

$$
\left[\begin{array}{cc}
\sigma_{1} I & \widetilde{X}_{1} \\
\widetilde{X}_{1}^{T} & \widetilde{P}
\end{array}\right] \geq 0, \quad\left[\begin{array}{cc}
\sigma_{2} I & \widetilde{X}_{1} \\
\widetilde{X}_{1}^{T} & \widetilde{Q}
\end{array}\right] \geq 0,\left[\begin{array}{cc}
\sigma_{3} I & \widetilde{X}_{1} \\
\widetilde{X}_{1}^{T} & \widetilde{R}
\end{array}\right] \geq 0 .
$$

and construct a feasibility problem, for given $h_{m}, \gamma, \kappa_{1}$ and $\kappa_{2}$, as follows:

$$
\begin{aligned}
& \text { Find } \bar{P}, \bar{Q}, \bar{R}, \widetilde{P}, \widetilde{Q}, \widetilde{R}, \widetilde{X}_{1}, X_{1}, \bar{N}_{1}, \bar{N}_{2}, S, W, Y_{c} \\
& \mu, \delta, \sigma_{i}, i=1,2,3 \\
& \text { subject to } \bar{P}>0, \bar{Q}>0, \bar{R}>0, \widetilde{P}>0, \widetilde{Q}>0, \widetilde{R}>0, \delta>0, \\
& \mu>0, \sigma_{i}>0, i=1,2,3,22 \text {, 23, 24, 35, 36, 37. }
\end{aligned}
$$

Then there exists an anti-windup gain $E_{c}$ leading to the maximization of the region of stability of the closed-loop system. It is noted that this problem still includes the nonlinear conditions 36 . However, using the idea introduced in [11], the feasibility problem in (38) can be converted to an iterative procedure involving LMI conditions:

$$
\begin{aligned}
& \text { Minimize Trace }\left(\bar{P} \widetilde{P}+\bar{Q} \widetilde{Q}+\bar{R} \widetilde{R}+\left(X_{1}+X_{1}^{T}\right)\left(\widetilde{X}_{1}+\widetilde{X}_{1}^{T}\right)\right) \\
& \text { subject to } \bar{P}>0, \bar{Q}>0, \bar{R}>0, \widetilde{P}>0, \widetilde{Q}>0, \widetilde{R}>0, \mu>0 \text {, } \\
& \delta>0, \sigma_{i}>0, i=1,2,3,22 \text {, 23, 24, 235, 37, } \\
& {\left[\begin{array}{ll}
\bar{P} & * \\
I & \widetilde{P}
\end{array}\right] \geq 0, \quad\left[\begin{array}{cc}
\bar{Q} & * \\
I & \widetilde{Q}
\end{array}\right] \geq 0, \quad\left[\begin{array}{cc}
\bar{R} & * \\
I & \widetilde{R}
\end{array}\right] \geq 0,} \\
& {\left[\begin{array}{cc}
X_{1}+X_{1}^{T} & * \\
I & \tilde{X}_{1}+\tilde{X}_{1}^{T}
\end{array}\right] \geq 0 .}
\end{aligned}
$$

This new LMIs problem can be solved by applying the cone complementarity algorithm [11] in the following manner:

Step 1 Given $h_{m}$ and choose a sufficiently large initial $\kappa_{1}=\kappa_{2}$. Find a set of feasible matrices $\left(\bar{P}, \bar{Q}, \bar{R}, W, X_{1}, S, Y_{c}, \widetilde{P}, \widetilde{Q}\right.$, $\left.\widetilde{R}, \widetilde{X}_{1}, \sigma_{i}, i=1,2,3\right)_{0}$ that satisfies 39 .

Step 2 Solve the following LMI minimization problem:

$$
\begin{aligned}
& \operatorname{Minimize} \\
& \operatorname{Trace}\left(\bar{P} \widetilde{P}_{0}+\bar{Q} \widetilde{Q}_{0}+\bar{R} \widetilde{R}_{0}+\left(X_{1}+X_{1}^{T}\right)\left(\widetilde{X}_{10}+\widetilde{X}_{10}^{T}\right)\right. \\
& \left.+\bar{P}_{0} \widetilde{P}+\bar{Q}_{0} \widetilde{Q}+\bar{R}_{0} \widetilde{R}+\left(X_{10}+X_{10}^{T}\right)\left(\widetilde{X}_{1}+\widetilde{X}_{1}^{T}\right)\right)
\end{aligned}
$$$$
\text { subject to LMIs in 39 }
$$

Step 3 Substitute the matrix variables from the previous step into 39): If the result is feasible, then set $E_{c}=Y_{c} S^{-1}$. If it is not feasible, then set the new matrices to be $\left(\bar{P}, \bar{Q}, \bar{R}, X_{1}, S\right.$, $\left.Y_{c}, W, \widetilde{P}, \widetilde{Q}, \widetilde{R}, \widetilde{X}_{1}, \sigma_{i}, i=1,2,3\right)_{0}$ and return to Step 2 .

Remark 2. The tuning scalar parameter $\alpha$ can be selected by applying a simple numerical optimization: see [6].

\section{NUMERICAL EXAMPLES}

In this section, we consider some examples to illustrate the feasibility and the effectiveness of the proposed design methodology. Example 1 The example is borrowed from [3]. Consider system [1], with $w(t)=0$ and the following parameters:

$$
\begin{aligned}
& A=\left[\begin{array}{ll}
1 & 0 \\
0 & 0
\end{array}\right], A_{h}=\left[\begin{array}{cc}
1 & 1.5 \\
0.3 & -2
\end{array}\right], B=\left[\begin{array}{c}
10 \\
0
\end{array}\right], \\
& C_{y}=\left[\begin{array}{ll}
5 & 1
\end{array}\right], u_{0}=15,
\end{aligned}
$$

The dynamic controller is given as:

$$
\begin{aligned}
& A_{c}=\left[\begin{array}{cc}
-20.2042 & 2.5216 \\
2.1415 & -4.4821
\end{array}\right], B_{c}=\left[\begin{array}{c}
1.9516 \\
-0.0649
\end{array}\right], \\
& C_{c}=\left[\begin{array}{ll}
-0.9165 & 0.1091
\end{array}\right], D_{c}=0
\end{aligned}
$$

By applying Theorem 1, with the numerical parameters obtained from the algorithm presented in section 4, with $\alpha=0.8$ and $d=0.1$, the stability of the system can be guaranteed with the static anti-windup gain $E_{c}=\left[\begin{array}{c}19.2296 \\ -67.6834\end{array}\right]$ for $h \leq 0.6467$ and $\sqrt{\kappa}=\sqrt{\kappa_{1}}=\sqrt{\kappa_{2}}=175.6059 * 10^{3}$.

The upper bound on the time-delay was found to be $h=0.4$ in [3] and [14] for the maximum radius 756.19 and $4.6355 * 10^{3}$, respectively. The obtained results are listed in Table 1, with a comparison of the maximum radius and the upper bound on the acceptable delay. Clearly, our result is less conservative than those of [3] and [14].

Table 1: Comparison of $h$ and maximum radius $\sqrt{\kappa}$
\begin{tabular}{|c|c|c|}
\hline & $\mathrm{h}$ & $\sqrt{\kappa}$ \\
\hline Gomes da Silva et al. [3] & 0.4 & 756.19 \\
\hline Wang et al. [14] & 0.4 & 4635.5 \\
\hline Theorem 1 in this paper & 0.6467 & 175605.9 \\
\hline
\end{tabular}

Example 2 Now, we subject the system in the previous example to actuator saturation and disturbances of the form (1), with

$$
B_{w}=\left[\begin{array}{l}
1 \\
0
\end{array}\right], C_{z}=\left[\begin{array}{ll}
0 & 1
\end{array}\right], D_{z}=0 .
$$

To apply the result in Theorem 1 we solve the optimization problem (32) with $d=0.1, \alpha=0.7$ and $h=0.6124$ obtaining the optimal value $\mu=0.0002$ and the static anti-windup gain $E_{c}=\left[\begin{array}{c}6.9491 \\ 28.3313\end{array}\right]$.

The $L_{2}$-gain can be determined from 33 , obtaining $\gamma=0.1$ with $\alpha=0.7, h=0.5833$ and $d=0.1$ for $\mu=1$ and the static antiwindup gain $E_{c}=\left[\begin{array}{c}25.3303 \\ -296.9706\end{array}\right]$.

\section{CONCLUSIONS}

The problem of anti-windup design for linear systems with timevarying delays and actuator saturation has been addressed. More precisely, using Lyapunov-Krasovskii functionals, we provide a methodology to compute an anti-windup compensator that ensures both $\mathcal{L}_{2}$ input-to-state stability and internal stability of the closedloop system. Provided that the maximal value of the time-varying delay and its derivative are known, convex optimization problems were proposed to compute the anti-windup gains aiming at three different control objectives. Some numerical examples have been presented to show the potential of the proposed methodology. 
As further work we can mention the derivation of simplification of the conditions obtain, the reduction of any remaining conservatism and the development of delay-independent conditions, adequate when the delay is not completely known.

\section{REFERENCES}

[1] J. M. Gomes da Silva Jr. and S. Tarbouriech. Local stabilization of discrete-time linear systems with saturating controls: an Imi-based approach. Eurographics Symposium on Rendering, pages 119-125, 2001.

[2] J. M. Gomes da Silva Jr. and S. Tarbouriech. Anti-windup design with guaranteed regions of stability: An lmi-based approach. IEEE Transactions on Automatic Control, 50:106$111,2005$.

[3] J. M. Gomes da Silva Jr., S. Tarbouriech, and G. Garcia. Using anti-windup for enlarging the stability of time-delay systems subject to input saturation. In In Proceedings of American Control Conference, Boston, Massachusetts, 2004.

[4] E. Delgado and A. Barreiro. Stability of teleoperation systems for time varying delays by lmi techniques. In Proceedings of the European Control Conference, Kos, Greece, 2007.

[5] F. El Haoussi and E. H. Tissir. Delay and its time-derivative dependent robust stability of uncertain neutral systems with saturating actuators. International Journal of Automation and Computing, 7:455-462, 2010.

[6] F. El Haoussi, E. H. Tissir, A. Hmamed, and F. Tadeo. Stabilization of neutral systems with saturating actuators. Journal of Control Science and Engineering, 1:1-9, 2011.

[7] F. El Haoussi, E. H. Tissir, H. Satori, and F. Tadeo. Robust stability analysis of teleoperation by delay-dependent neutral lmi techniques. Applied Mathematical Sciences, 8:26872700, 2014.

[8] F. El Haoussi, E. H. Tissir, F. Tadeo, and A. Hmamed. Delaydependent stabilisation of systems with time-delayed state and control: application to a quadruple-tank process,. International Journal of Systems Science, 42:41-49, 2011.

[9] Y. He, M. Wu, J. H. She, and G. P. Liu. Parameter-dependent lyapunov functional for stability of time-delay systems with polytopic-type uncertainties. IEEE Transactions on Automatic Control, 49:828-832, 2004.

[10] T. Hu and Z. Lin. Control systems with actuator saturation: Analysis and design. In Boston, MA: Birkhauser, 2001.

[11] Y. S. Lee, Y. S. Moon, W. H. Kwon, and P. G. Park. Delaydependent robust h1 control for uncertain systems with a state-delay. Automatica, 40:65-72, 2004.

[12] Z. Lin and A. Saberi. Semi-global exponential stabilization of linear systems subject to input saturation via linear feedbacks. Systems and Control Letters, 21:225-239, 1993.

[13] S. Tarbouriech, J. M. Gomes da Silva Jr., and G. Garcia. Delay-dependent anti-windup strategy for linear systems with saturating inputs and delayed outputs. International Journal of Robust and Nonlinear Control, 14:665-682, 2004.

[14] Y. Wang, Y. Cao, and Y. Sun. Anti-windup compensator gain design for time-delay systems with constraints. Acta Automatica Sinica, 32:455-470, 2006.

[15] S. Xu and J. Lam. A survey of linear matrix inequality techniques in stability analysis of delay systems. International Journal of Systems Science, 39:1095-1113, 2008. 